\title{
ECG Signal Classification using DD-CWT and RFC
}

\author{
N. N. S. V. Rama Raju1)
}

\begin{abstract}
The Fundamental problem in heart failure is the inability of the heart to the metabolic demands of the body. Acute heart failure or decompensated heart failure is the condition in which the heart is not able to pump the blood effectively. The Body has compensated mechanisms to maintain homeostasis. It leads to an increase in sympathetic activity that causes increased cardiac output. This paper presents a technique for the automated classification of cardiopathies from AN EKG (ECG) with DD-CWT based mostly f feature extraction and random forest-based classification for various cardiopathies. These parameters area unit calculated for beats with four varieties of abnormalities from graphical record records retrieved from the MIT-BIH heart condition information. This study resulted in an exceedingly most overall rate of $96.14 \%$ for all parameters.
\end{abstract}

Keywords : ECG, DD-CWT, Random, forest, MIT-BIH

\section{Introduction}

The classification of different types of heart disease from electrocardiogram (ECG) is of major importance in the diagnosis of cardiac dysfunctions[1-5]. The automation of this analysis is nowadays a well-established practice that can ensure a high rate in knowledge of cardiac abnormalities. Different parameterization techniques, time and frequency, were tested in particular measurements related to morphological parameters characterizing ECG events such as amplitudes, surfaces intervals and slopes in the QRS complex. Most of these studies are limited to a limited number of arrhythmia cardiopathies, particularly normal beating and premature ventricular contraction (PVC)[4].

In this article, we propose to develop a classification system of 8 arrhythmias based on hidden Markov models. The arrhythmias considered are normal rhythm (NOR); ventricular premature contraction (PVC); the left branch block (LBBB); (RBBB), a trial premature beat (AP), merging of ventricular and normal beats, pulse beat $(\mathrm{P})$, and fusion of normal and trained beats.

Received(July 25, 2019), Review Result(1st: August 30, 2019, 2nd: October 24, 2019), Accepted(November 15, 2019)

1) (Research Scholar) Department of Electronics and Communications Engineering, GITAM University, Visakhapatnam, AP, India email: ramarajupro@gmail.com 
An ECG example figure shown in [Fig. 1] which will represent the pulse of a human being in the form of ups and downs in the figure as high's and low's[4].
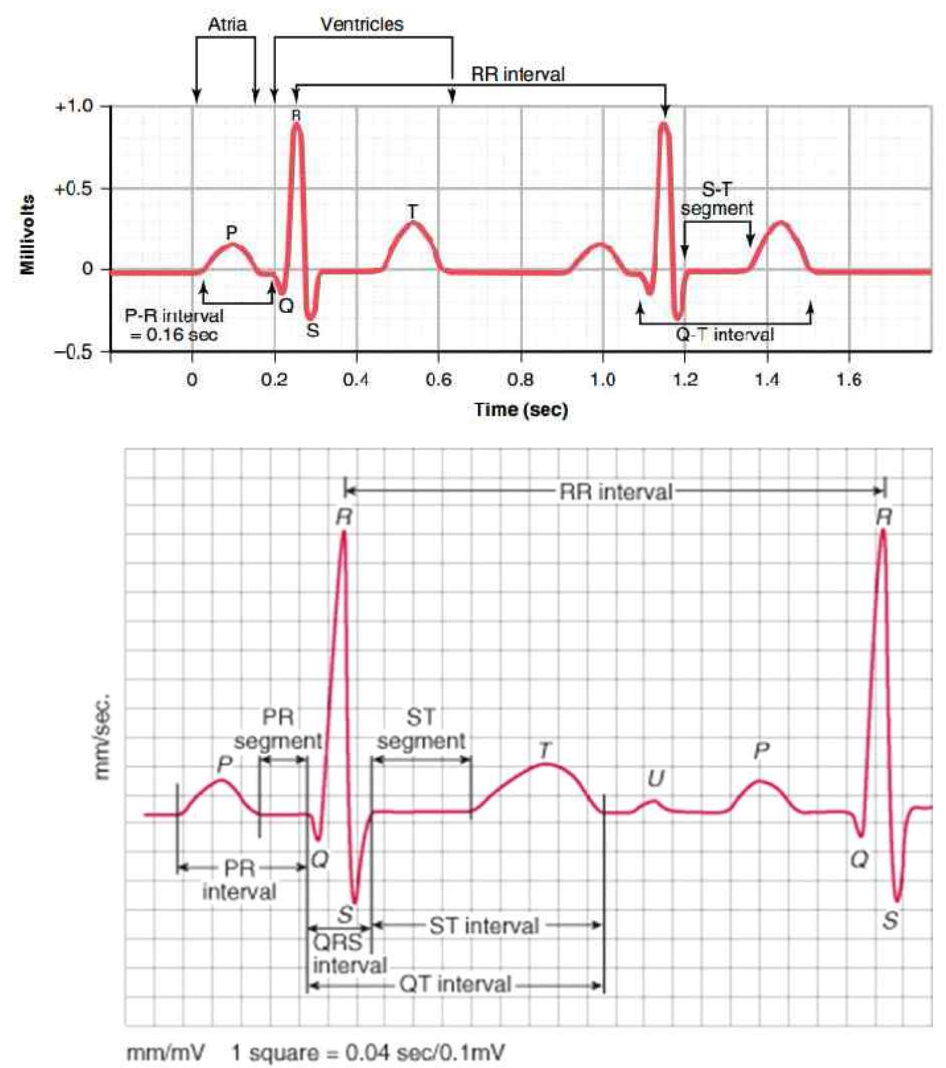

[Fig. 1] Components of a Normal Electrocardiogram (ECG)[1]

Every ECG gives two sorts of data: One that is given by the term of the electrical wave that goes through the heart and decides if the electrical action is ordinary, moderate or unpredictable; and another is the measure of electrical movement that goes through the heart muscle demonstrating which parts of the heart are too huge or if there is a work over-burden[5].

\section{State of the Art}

The Wavelet Transform analysis is being connected to a wide assortment of a biomedical signals containing electromyographic (EMG) signals, electroencephalographic (EEG) signals, clinical sounds, respiratory patterns, blood pressure trends, and deoxyribonucleic acid sequences 
(DNA), along with the signals object of this project the electrocardiographic (ECG).

At present, there are a large number of applications for the processing of signals of physiological origin, mainly due to the complexity in the extraction of rules and specific characteristics for the implementation of algorithms that unequivocally reflect the medical knowledge derived from the interpretation of the biological/biomedical signals treated[6].

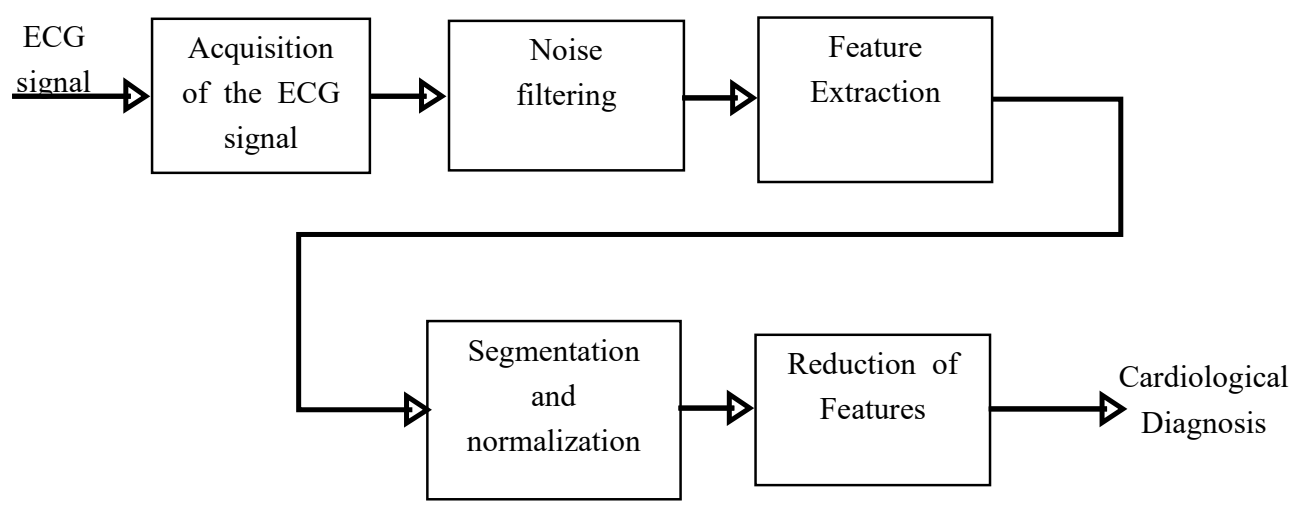

[Fig. 2] General Scenario Image in ECG

\section{Methodology}

The methodology followed is divided into several stages:

\subsection{Obtaining ECG Signals}

It starts, as in the previous cases, by selecting the ECG signals to use. In this case, 13 ECG signals have been taken from as many patients at the base hospital or at any laboratory[7].

\subsection{Selection of Wavelet}

The utilization of the Wavelet Transform has picked up large acceptance in the time-frequency analysis on account of the adaptability offer the functions of scale, which makes it necessary to select the family appropriately.

Assortment of the wavelet family is an essential duty before processes of recognition of singularities. Usually, one or several Wavelet is selected that resemble the signal to be analyzed.

Among the Wavelets families like the Haar family, Biorthogonal, Coiflets, Daubechies, Meyer, 
Morlet, Symlets, Sombrero Mexicano, and other complex Wavelets, we proceed to choose the DaubechiesWavelet (Db8)[8-11].

This Wavelet demonstrates an extraordinary closeness with the thought of the low-frequency reason for which has been chosen for the extraction of characteristics of the ECG signal[12].

\subsection{Preprocessing}

The ECG signal contains various types of noise produced by different agents: frequency interference, reference drift, electrodes, polarization noise, muscle noise, internal amplifier, from the movement of the electrodes, etc. One of the problems common in the processing of ECG signals is that of the baseline that goes through the elimination and suppression of noise, a fact that is discussed below. Elimination of reference drift or baseline

The lack of a baseline is related to the noise caused by the devices in the acquisition and analog-digital conversion of the ECG signal. For, Therefore, it is necessary to eliminate the lack of baseline in the exploration of the ECG to diminish modifications in the morphology of the heartbeat without a physiological counterpart.

Breathing and impedance changes of the electrodes due to the transpiration are essential causes of baseline variability in most of the different types of ECG signal records. This elimination of the reference drift is highly demanded by cardiologists.

The spectral content of the baseline is very and is generally in a range much below $0.5 \mathrm{~Hz}$. It eliminates the baseline and signals noise ECG so that the ECG signal has a baseline at zero millivolts.

The drift of the baseline is removed without fluctuating or disrupting the features of the ECG waveform by using a bandpass filter @ 0.5 - $40 \mathrm{~Hz}$.

As well as the dominant frequency in the frequency range of the ECG signal is close to 40 $\mathrm{Hz}$ the upper ones are eliminated in order to suppress the high frequency noise signal[12]. This stage is implemented with a bandpass filter (Bandpass -BP-) IIR Butterworth with a cutoff frequency of less than $\mathrm{fL}=0.5 \mathrm{~Hz}$ and frequently of uppercut $\mathrm{fH}=40 \mathrm{~Hz}$. The transfer function is represented by $\mathrm{HH}$ :

$$
\begin{gathered}
H(z)=\frac{B(z)}{A(z)} \\
B(z)=z^{10}-5 z^{8}+10 z^{6}-10 z^{4}+5 z^{2}-1
\end{gathered}
$$




\section{Wavelet Transform Thresholding}

In non-stationary signals where the use of a Digital filter or adaptive algorithms for the possible loss of information, we use the Discrete Wavelet Transform[12]. The ECG signal is broken down into several subbands by the application of the Discrete Wavelet Transform (DWT) and by applying the Wavelet that best adapts. Subsequently, the Wavelet coefficients are modified by the application of a threshold function, and then, with the inverse transform (IDWT) the signal is reconstructed without noise.

As the detailed coefficients in the minor scale have vanished from the actual signal, the high-frequency part of the ECG signal reduces. With this elimination, smoothness comes into the signal, and the noise vanishes. It is because of the fact that the high-frequency components characterize the noise.

High frequency components have been added along the tracks of transmission. The DWT implicitly contributes to the ECG signal noise.

The elimination method is applied to the Wavelet coefficients (Wavelet thresholding) and after applying the thresholds SURE (soft), with the decomposition Multiresolution using the Wavelet Daubechies of order 8 (Db8) with 8 scales of decomposition.

\subsection{R Wave and QRS Complex Detection}

The majority of the energy in the QRS complex concentrates on the levels of decomposition 23-25. The QRS complex model can be represented as,

$$
e_{1}=d_{3}+d_{4}+d_{5}
$$

This expression correctly configures the QRS region, however it is challenging to recognize the e_2 function as,

$$
e_{2}=\frac{d_{4}}{2^{n}}\left(d_{3}+d_{5}\right)
$$

The module has the signals e_1 ande_2 as,

$$
e 3=\left|e_{1} \times e_{2}\right|
$$




\subsection{Feature Extraction}

Wavelets have found a very large number of applications in the medical field, mainly in the analysis of bio-acoustic, electroencephalographic, and medical imaging signals. In the field of cardiology, wavelets have been used to solve various problems of detection of waves, fiducial points and the presence of PT.

\section{Descriptive Studies}

The first work on the application of wavelets to the ECG signal was mainly descriptive. The Meste et al. 1989 was one of the first to apply the wavelet transformation to ECGs recorded by a digital acquisition system with high resolution $(5 \mathrm{kHz})$. Wavelet transformation of the ECG proved to be interesting. Nevertheless, the contribution of time-scale representations of the ECG must be evaluated. A quantitative comparison in terms of the diagnostic performance of these methods was therefore necessary. For this purpose, the authors sought to define parameters making it possible to quantify the information contained in the time-frequency representations. Dickhaus in 1992 proposed to use as a quantizer the volume of time-scale representation of the Morlet transformation between frequencies 100 and $300 \mathrm{~Hz}$ and over a $300 \mathrm{~ms}$ interval centered on the QRS complex. With this parameter, Dickhaus obtained significantly different values $(p=0.0005$, Wilcoxon test) between its test population consisting of 21 patients with sustained TV and its reference population consisting of 29 subjects healthy. Patients were not on antiarrhythmic therapy and none had BB. Always using the same parameter type but with time-frequency zones.

\subsection{Double Density}

Because of multiplying the number of wavelets at each progression, the proposed approach has a bigger calculation time contrasted with DWT. The calculation was redesigned in many structures from 1-D and 2-D up to double density complexs. The execution of DDWT is controlled with the oversampled investigation by the use of a synthesis filters bank (see [Fig. 3]). The given wavelets comprises a frequency reaction (see [Fig. 4]) for $\mathrm{h}^{\wedge} 0, \mathrm{~h}^{\wedge} 1$, and $\mathrm{h}^{\wedge} 2$ filters. The primary filter is low pass and rest of the two are high pass. The synthesis banks fuse its low pass fragment for oversampling and the tedious procedure makes an oversampled filter bank. This engineering can be viewed as the replacement of multi-resolution architecture (MRA) having scaling function $(\varnothing)$ for $\phi^{\wedge} 1$ and $\phi^{\wedge} 2$ wavelets. 


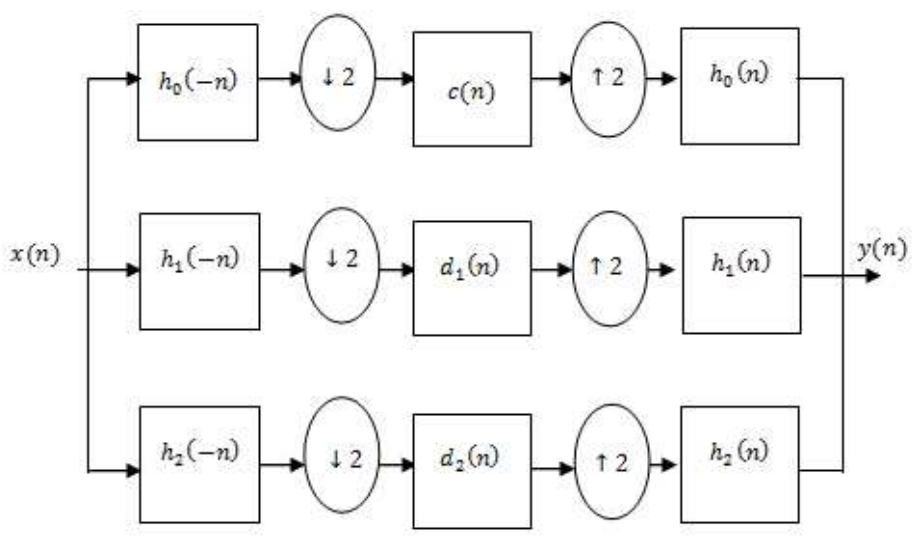

[Fig. 3] Oversampled Synthesis Filter Bank

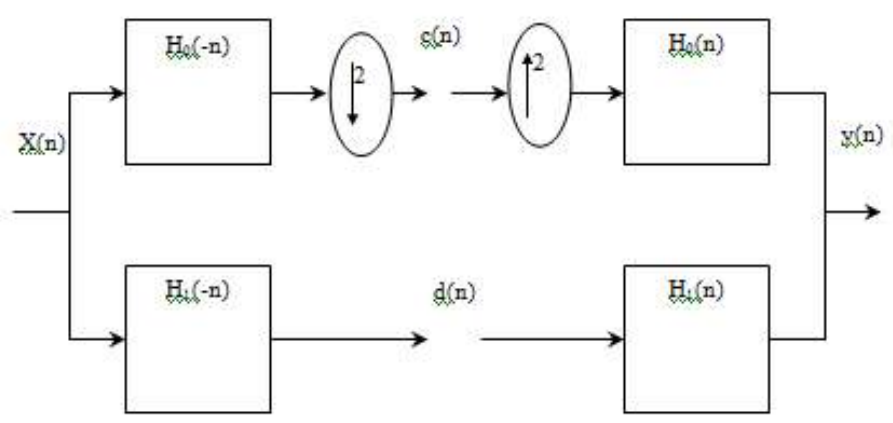

[Fig. 4] Illustration of Filter Bank Block

[Fig. 4] shows an oversampled filter bank having low and high pass filters which is utilized to construct an arrangement of DD-DWT.

The connection between input and output is explained as:

$$
\begin{aligned}
& Y(z)=\frac{1}{2}\left[H_{0}(z) H_{0}\left(\frac{1}{z}\right)+H_{1}(z) H_{1}\left(\frac{1}{z}\right)+H_{2}(z) H_{2}\left(\frac{1}{z}\right)\right] X(z)+ \\
& =\frac{1}{2}\left[H_{0}(z) H_{0}\left(-\frac{1}{z}\right)+H_{1}(z) H_{1}\left(-\frac{1}{z}\right)+H_{2}(z) H_{2}\left(-\frac{1}{z}\right)\right] X(-z)
\end{aligned}
$$

The filter bank utilized to assemble the DD-DWT looks like the wavelet outline with $\phi(t)$ as 
scaling function and $\Psi \_1(t)$ and $\Psi \_2(t)$ wavelets. As per the reports of dyadic wavelet space:

$$
\begin{aligned}
& V_{j}=\operatorname{Span}_{n \in Z}\left\{\phi\left(2^{j} t-n\right)\right\} \\
& V_{j}=\operatorname{Span}_{n \in Z}\left\{\phi\left(2^{j} t-n\right)\right\}
\end{aligned}
$$

The structure of a dyadic wavelet is characterized by a single wavelet $\psi$ and scaling function $\phi$. The extra wavelet in equation (9) avoids any possibility of deficiency (incompleteness). The scaling function and wavelets satisfy the equations.

$$
\begin{gathered}
\varnothing(t)=\sqrt{2} \sum_{n} h_{0}(n) \varnothing(2 t-n) \\
\psi_{i}(t)=\sqrt{2} \sum_{n} h_{i}(n) \varnothing(2 t-n) \quad i=1,2 \ldots
\end{gathered}
$$

\section{Experimental Setup}

\section{Database 1: MIT-BIH Normal Sinus Rhythm Database}

In order to verify the performance of the model considered, an experimental setup had been considered and modeled. The capacity of the considered model can generate 18 full ECG reports of around 18 patients for whom no important arrhythmia will exist. The horizontal axis is used to represent either the time or the velocity of the model to be measured and the vertical axis will be used for measuring either the voltage or the amplitude of the reports. The identification of several factors or the components like the ST segment, $\mathrm{U}$ wave, P wave, QRS complex etc. The $\mathrm{P}$ wave usually has the values which were at a peak not less than $0.25 \mathrm{~m}$ Voltage and 0.11 seconds which may be less than or equal in duration. The interval between the waves collected or selected from the list like the Q-T waves may represent either the depolarization or the repolarization of the time and can be measured from the $Q$ wave starting points or the wave at $\mathrm{Q}$ or sometimes it may be at the end of the wave $\mathrm{T}$. These values will vary with the time values and heart rate values also. This time slot may vary from 0.35 seconds to 0.42 seconds and also the heart beating rate of the patients may vary from the values like the 60 to 90 beats per minute on an average. 


\begin{tabular}{|c|c|c|}
\hline & Proposed & DWT and Random Forest \\
\hline Normal & 99.3 & 98.2 \\
\hline RBBB & 98.4 & 97.8 \\
\hline APC & 95.8 & 94.5 \\
\hline PVC & 94.3 & 91.3 \\
\hline LBBB & 98.2 & 97.8 \\
\hline
\end{tabular}

\section{Conclusion}

The work conferred during this paper consists of the main of 2 domains: signal process and applied math learning. Signal process techniques have allowed North American countries to phase. We have a tendency to create a choice rule that permits North American countries to differentiate associate degree position beat from a traditional beat. To do this, we have a tendency to select to figure with the SVMs technique, an evidenced formula. These pre-treatments are performed within the time-scale domain as a result of the signals suffering from non-stationary, notably throughout the cardiac arrhythmia phases. The detected QRS is used as a feature extraction by victimization Double Density twin Tree complicated ripple remodel (DD-DT-CWT).These options area unit trained with random forest classifier and accuracy achieved is $96.5 \%$.

\section{References}

[1] Myeong-Kil Song and Kwang-Min Park, Design and Implementation of a 9V Mini-Electrocardiograph (ECG) System, Journal of the Korea Academia-Industrial Cooperation Society, (2008), Vol.9, No.5 pp.1130-1133, UCI: G704-001653.2008.9.5.039

[2] Gye Rok Jeon, Dong Keun Jung, Gi Ryun Kim and Bum Joo Shin, The Development of Integrated Sensor System for Measuring Simultaneously ECG, PPG and PPW, Journal of the Korea Academia-Industrial Cooperation Society, (2009), Vol.10, No.5 pp.992-999, UCI: G704-001653.2009.10.5.009

[3] Se-Jong Ahn, Chang Joo Lim, Yong-Gwon Kim and Sung-Taek Chung, Study on R-peak Detection Algorithm of Arrhythmia Patients in ECG, Journal of the Korea Academia-Industrial Cooperation Society, (2011), Vol.12, No.10 pp.4443-4449, UCI: G704-001653.2011.12.10.047

[4] K. V. L. Narayana, A. B. Rao, Wavelet based QRS detection in ECG using MATLAB, Innovative Systems Design and Engineering, (2011), Vol.2, No.7, pp.60-69.

[5] J. E. Hall, Guyton and Hall Textbook of Medical Physiology E-Book, Elsevier Health Sciences, (2015) 
[6] C. Saritha, V. Sukanya, and Y. N. Murthy, ECG signal analysis using wavelet transforms, Bulgarian Journal of Physics, (2008), Vol.35, pp.68-77.

[7] P. Sasikala, and R. S. D. WahidaBanu, Extraction of $\mathrm{P}$ wave and $\mathrm{T}$ wave in Electrocardiogram using Wavelet Transform, International Journal of Computer Science and Information Technologies, (2011), Vol.2, No.1, pp.489-493.

[8] S. Behbahani, N. J. Dabanloo, Detection of QRS complexes in the ECG signal using multiresolution wavelet and thresholding method, 2011 Computing in Cardiology, (2011), September 18-21; Hangzhou, China.

[9] Z. Zidelmal, A. Amirou, M. Adnane, and A. Belouchrani, QRS detection based on wavelet coefficients, Computer Methods and Programs in Biomedicine, (2012), Vol.107, No.3, pp.490-496, DOI: https://doi.org/10.1016/j.cmpb.2011.12.004

[10] M. Thomas, M. K. Das, and S. Ari, Automatic ECG arrhythmia classification using dual tree complex wavelet based features, AEU-International Journal of Electronics and Communications, (2015), Vol.69, No.4, pp.715-721, DOI: https://doi.org/10.1016/j.aeue.2014.12.013

[11] P. S. Addison, Wavelet transforms and the ECG: a review, Physiological measurement, (2005), Vol.26, No.5, pp.R155-R199, DOI: 10.1088/0967-3334/26/5/R01

[12] M. A. Kabir, and C. Shahnaz, Denoising of ECG signals based on noise reduction algorithms in EMD and wavelet domains, Biomedical Signal Processing and Control, (2012), Vol.7, No.5, pp.481-489, DOI: https://doi.org/10.1016/j.bspc.2011.11.003 